\title{
Anxiogenic Effect of Low-dose Methamphetamine in the Test of Elevated Plus-maze
}

\author{
Pometlová M., Nohejlová-Deykun K., Šlamberová R. \\ Department of Normal, Pathological and Clinical Physiology, Third Faculty \\ of Medicine, Charles University in Prague, Prague, Czech Republic \\ Received February 23, 2012; Accepted June 25, 2012.
}

Key words: Anxiety - Behavior - Elevated plus-maze - Methamphetamine

Abstract: Methamphetamines (MA) are psychostimulant drugs that are known to change individuals' behavior. Psychostimulants could either evoke positive emotions (e.g. joy and happiness) or attenuate negative emotional states (e.g. anxiety and depression) in humans. In animal experiments, the test of elevated plus-maze (EPM) is widely used.This test is appropriate for evaluation of anxiolytic and anxiogenic drug effects, or for examination of specific subtypes of anxiety disorders. The aim of the present study was to examine the effect of acute single dose of MA $(1 \mathrm{mg} / \mathrm{kg})$ on the behavior of laboratory rat in the EPM.The detailed ethologic analysis of behavior was performed using a modified protocol based on the study of Fernández Espejo (1997). Our results demonstrated that MA affects rat's behavior in the EPM in the majority of analyzed categories. The present protocol allowed us to determine positive anxiogenic effect of MA.

This study was supported by grant GA P303/10/0580 from Grant Agency of the Czech Republic, project CSM 110/2012 from Ministry of Education, Youth and Sports and project 264706/SVV/2012 from Charles University in Prague.

Mailing Address: Marie Pometlová, MD., PhD., Department of Normal, Pathological and Clinical Physiology, Third Faculty of Medicine, Charles University in Prague, Ke Karlovu 4, 12000 Prague 2, Czech Republic; Phone: +420 224902 725; Fax: +420 224923 827; e-mail: mpomet@If3.cuni.cz 


\section{Introduction}

Psychostimulants, including methamphetamine (MA), influence individual behavior. In humans, psychostimulants induce feeling of pleasure and happiness or suppress negative states as anxiety and depression (Nesse and Berridge, 1997). The effects of the particular drug depend on its chemical structure and its interaction with positive or negative emotion circuits of the CNS. At the cellular level psychostimulants activate molecular signalization in dopaminergic and glutamatergic CNS systems. Both of those systems are widely distributed in many areas of the cerebral cortex and the basal ganglia; and play key role in integration of motor activity, motivation, learning and memory. They also optimize adaptation reactions of the organism to the environment with respect of food acquisition or avoidance of danger (Kelley, 2004). Many of misused substances, including psychostimulants, primary affect the above mentioned circuits and are able to induce long-term changes, which would alter behaviors and would lead to maladaptation (Koob and Le Moal, 1997; Hyman and Malenka, 2001; Kelley and Berridge, 2002).

In the present experimental work, in order to evaluate behavioral changes of laboratory rats induced by MA, elevated plus-maze test (EPM) was used. The EPM is the most commonly used for examination of effects of anxiolytic or anxiogenic agents; alternatively it is used to test individual subtypes of anxiogenic disorders (Hogg, 1996). EPM tests may also be applied for better understanding of biological basis of emotions in the respect of pain, drug addiction and withdrawal syndrome (File, 1993; Carobrez and Bertoglio, 2005).

The ethologic evaluation of laboratory animals' behavior in the EPM varies in studies of different authors (Gerlai et al., 2006). In the present work the scheme of evaluation described by Fernández Espejo (1997) was used. In this scheme animal's behavior is divided into four categories. The first category includes the behavior related to anxiety. The anxiolytic behavior is described as activities in open arms, and anxiogenic behavior involves activities in the closed arms and the center. The second behavioral category in the EPM evaluates motor activity. The third category describes approach-avoid conflict and also depicts other types of behavior in respect to open and closed arms, as for example, positioning on the end of the open arm or return (retrieval) to the closed arm. The last category includes displacement behavior, in the terms of inadequate to the conditions behavior, as grooming. The precise description of individual types of behavior is described in methods section.

It was proved by the Markovian sequence analysis that the main behavioral pattern of rodents in the EPM is sniffing, which gives raise to the rest of the activities. The sniffing is included to the motor behavior category. An anxiety is closely related only to the behavior in the EPM described in the first category. In contrast, behaviors described in the third category are anxiety-independent behavioral patterns. They reflect only dimension of approach-avoid conflict (Fernández Espejo, 1997). 
The decrease of social interactions in the tests of social behavior of laboratory rats after the application of low doses of MA $(0.5,1.0$ and $1.5 \mathrm{mg} / \mathrm{kg})$ was described in our previous experimental works (Pometlová et al., 2007; Slamberova et al., 2010). This effect of MA was considered to be anxiogenic (File and Hyde, 1978). However, the following work (Pometlová et al., 2008) did not confirm the expected anxiogenic effect of MA in EPM tests of anxiety. Nevertheless, there were found certain differences of behavior in comparison to control animals. Therefore, in the present work the MA effects on behavior were re-tested and modified experimental protocol was used with detailed analysis of behavioral patterns.

\section{Material and Methods}

Adult Wistar male rats were divided into two groups ( $n=8, A n L a b)$. To the experimental group of animals subcutaneous MA in the dose of $1 \mathrm{mg} / \mathrm{kg}$ was applied, while control group received saline subcutaneously. All of the animals were handled according to the protocol described in Behavioral Science Protocols (Geyer and Swerdlow, 2007) during three days prior to the EPM tests. Applications of MA or saline were the same as described in the previous works (Pometlová et al., 2007, 2008), thirty minutes prior to the test of behavior in the EPM. All the testing was conducted between 9:00 a.m. and 12:00 p.m.

\section{EPM test}

The dark plastic plus maze was positioned $50 \mathrm{~cm}$ above the floor. The sides of closed arms were $40 \mathrm{~cm}$ high, of open arms $1 \mathrm{~cm}$ high in order to prevent animals slip and fall from the arms. All the arms were $10 \mathrm{~cm}$ wide with the center square $10 \times 10 \mathrm{~cm}$. Each animal was positioned to the center square with a nose aiming to one of the closed arm. Animals' behavior in the EPM was video-recorded during five minutes. The surface of the maze was cleaned with $20 \%$ ethyl alcohol and dried in between the individual testing.

\section{Ethologic evaluation of behavior}

Acquired video records were evaluated with use of ODLog program (Macropod Software). The modified ethologic evaluation protocol was used as in the study of Fernández Espejo (1997). The number of entries to the open arms and total time spent there was considered as anxiolytic behavior. Head-dipping (Dip) and stretched attend posture (SAP) were considered as anxiogenic behavior. Dip was defined as head-dipping that occurred on the center square or the closed arm, when the body of an animal was remaining in the closed arm or central square. SAP was recorded when it was occurring on a closed arm or center square. Motor behavior was described by the following activities: sniffing, number of entrances to the closed arms and total number of entrances to all of the arms. In the category approach-avoid conflict was evaluated number of returns to the closed arm 
(avoidance). The last category of displacement behavior could not be evaluated because it did not occur at low doses of MA used in the present study.

As anxiogenic behavior was considered (Dip) and stretched attend posture occurring on a closed arm or center square (SAP).

\section{Statistical analysis}

Data were tested for normality of distribution using the Kolmogorov-Smirnov test. One-way ANOVA and Mann-Whitney tests were used as appropriate (GraphPad Prism ${ }^{\circledR}$, USA). Differences were considered significant if $p<0.05$.

Experiments were conducted according to the guidelines of the Ministry of Health of the Czech Republic. The animal protocols were approved by the Ethics committee of the Third Faculty of Medicine, Charles University in Prague.

\section{Results}

Anxiogenic behavior

Number of entrances to open arms: MA group animals were entering open arms less frequently as control (saline) animals $(F(1,14)=6.54 ; p<0.05$, Figure 1$)$. There were no differences in total time spent in open arms between experimental and control animals.

Head-dipping (Dip): MA group animals did less head-dippings that control group; however difference did not show to be significant.

\section{Motor activity}

Total number of entrances to all of the arms: There was decreased number of total entrances to all the arms in MA animals when comparing the control $(F(1,14)$ $=7.63 ; p<0.05$, Figure 2). This activity did not change during five minutes of test record.

Number of entrances to the closed arms: There were no differences in the number of closed arm entrances between groups. However, the number decreased with the time (5 minutes) of recording in both, $M A$ and saline groups $(F(1,14)=2.99 ; p<0.05)$

Sniffing: MA group showed increased number of sniffing episodes when compared to the controls $(F(1,14)=37.91 ; p<0.001$, Figure $3 A)$. In contrast, total time spent by sniffing in MA group was significantly shorter $(F(1,14)=6.59 ; p<0.05$, Figure 3B).

\section{Approach-avoid conflict}

Number of returns to the closed arm: MA group animals had higher number of returns to the closed arms than control animals $(F(1,14)=7.03 ; p<0.01$, Figure 4).

\section{Discussion}

The MA caused behavioral changes of laboratory rats in all evaluated categories, although, the effect differed in some of the observed activities. For example, 


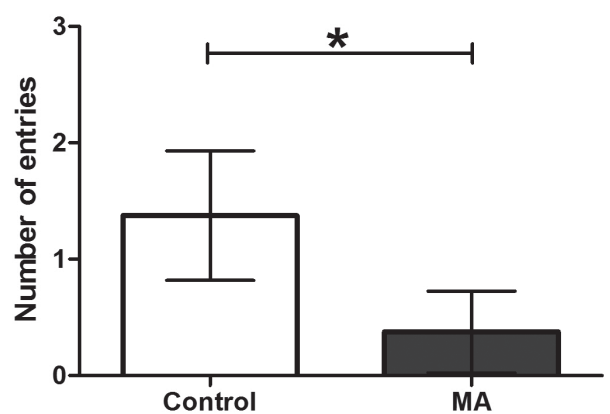

Figure 1 - Number of entries to open arms. MA decreased the number of entrances. Data present mean $\pm S E M, * p<0.05$.

A

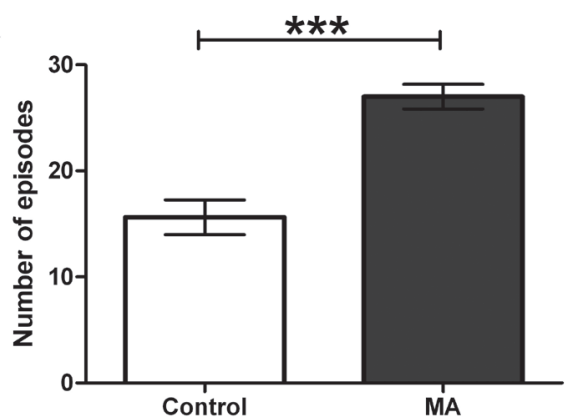

B

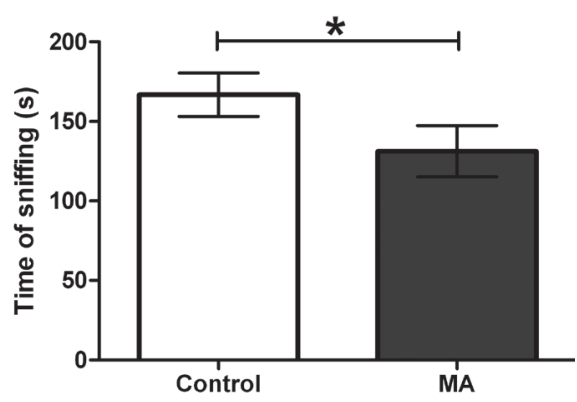

Figure 3 - Sniffing. Graph A depicts number of sniffing episodes that was higher in MA group. Graph $B$ shows total time spent by sniffing, which, in contrast, was grater in control group. Data present mean $\pm S E M$, $* * * p \leq 0.001, * p<0.05$.

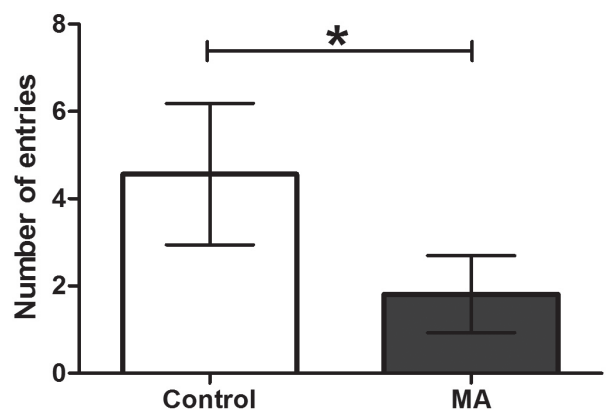

Figure 2 - Total number of entrances to all of the arms. MA decreased the number of entrances. Data present mean $\pm S E M, * p<0.05$.

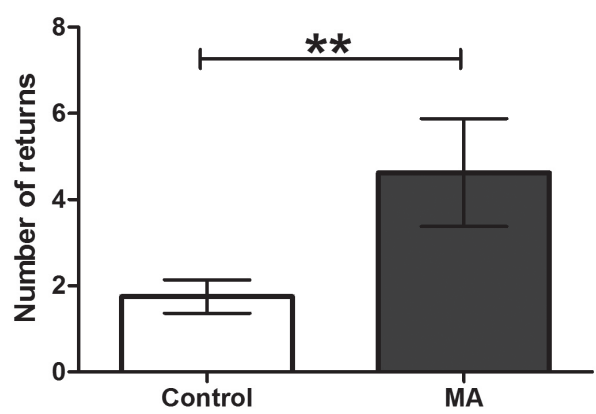

Figure 4 - Number of returns to the closed arms. MA significantly increased the number of returns to the closed arms. Data present mean \pm SEM, $* * p<0.01$. 
assessment of anxiogenic behavior showed significant decrease in the number of open arm entrances. However, the rest of parameters, such as Dip in the central square or SAP, that are also considered as anxiogenic behavior, did not differ from control animals. Moreover, head-dipping parameter showed to have tendency of anxiolytic pattern of behavior. Therefore, with use of ethological evaluation, it is not possible determine without doubt the effect of acute low-dose MA as anxiogenic. When the number of entrances to open arms was compared with total locomotion that is determined by number of entrances to all of the arms, it is clear that MA animals had lower percentage of entrances to the open arms (10.3\%) than control animals (20.5\%). The percentage of time spent in the open arms has similar tendency ( $\mathrm{MA}=0.1 \%$ and control $=4.7 \%$ ). When taking those findings into the account, the decreased number of open arm entrances can be accepted as clear anxiogenic effect of acute single low dose of MA (1 mg/kg). This conclusion is in agreement with the literature, where anxiety is induced by MA and other psychostimulants, though these authors use higher doses of psychostimulants and repeated administration (Hayase et al., 2006; Biala and Kruk, 2007). In our previous work (Pometlová et al., 2008; Slamberova et al., 2010) the anxiogenic effect of MA was not confirmed either by presence of stretched attend posture or by changes in number of open arm entrances. These differences in our previous and present work are probably caused by more precise evaluation of EPM test findings by modified ethologic evaluation protocol. Animal handling prior the experiment also play role in changes of the EPM test outcome (Hogg, 1996). It proves again that EPM test has a number of procedural limitations (Carobrez and Bertoglio, 2005).

The most remarkable changes were found in the category of motor behavior, i.e. total number of entrances to all of the arms and sniffing. The total number of entrances describes non-specific stimulation of locomotion - arousal/locomotor (Weiss et al., 1998). Increased locomotion in general could be confusing finding during evaluation of anxiolytic or anxiogenic effect (Rodgers et al., 1997; Weiss et al., 1998). It was demonstrated that MA decreases activity presented by total number of entrances to the all arms of the EPM, which reflected decreased number of entrances to open arms but there was no changes in the number of closed arms entrances in respect to control animals. There were also no differences in exploratory activity in the EPM of either group in our previous work (Pometlová et al., 2008). These results are in agreement with findings showing increased activity only in animals with prenatal application of MA but not in controls in the open field test (Bubenikova-Valesova et al., 2009). However, in the test of social interaction there was increase of locomotion after single low-dose MA application (Pometlová et al., 2007; Slamberova et al., 2010). Therefore, manifestations of changes in general motor activity cased by single low-dose MA are dependent on the test environment. This is well known fact that reflects MA effect (Hassler and Wagner, 1975). 
The sniffing presents normal exploratory behavior of rodents (Blanchard and Blanchard, 1999). According to Fernández Espejo (1997) it is a typical behavior in the EPM.

The MA and control groups were significantly different in this type of behavior. MA markedly increased frequency of sniffing episodes, while shortening their duration, i.e. many short episodes were observed. In contrast, control animals were sniffing less frequently but had longer duration of each episode. For psychostimulant-induced stereotypy in rodents, continuous sniffing is typical. But behavior of animals from the present study is in conflict with the described stereotypic sniffing induced by MA (Tatsuta et al., 2005). Never the less, it is worth to mention that authors had used doses of MA ten times higher (Tatsuta et al., 2005; Kitanaka et al., 2007) than was used in the present work.

In the category of approach-avoid conflict, the increased number of closed arm returns was observed, i.e. the negative part of the conflict - avoidance. Assuming that evaluation of anxiety level is based on conflict of avoidance of open space and exploration (Pellow et al., 1985), our findings confirm anxiogenic effect of MA at the used dose. Even though, according to Markovian sequence analysis should not be dependent on anxiety (Fernández Espejo, 1997).

\section{Conclusion}

The laboratory rats' behavior in the EPM after handling was evaluated by modified method which was introduced by Fernández Espejo (1997). It was shown that single low-dose of MA leads to behavioral changes in all evaluated categories. The most remarkable changes were observed in the category of motor behavior, e.g. sniffing episodes were shorter but with increased frequency. It could be concluded that a single low-dose of MA, which does not evoke stereotypy, changes some of basic constituents of laboratory rats' natural behavior. In addition, an anxiogenic effect of MA in terms of decreased visiting the open arms of the EPM was also found.

\section{References}

Biala, G., Kruk, M. (2007) Amphetamine-induced anxiety-related behavior in animal models. Pharmacol. Rep. 59(6), 636-644.

Blanchard, D. C., Blanchard, R. J. (1999) Cocaine potentiates defensive behaviors related to fear and anxiety. Neurosci. Biobehav. Rev. 23(7), 981-991.

Bubenikova-Valesova, V., Kacer, P., Syslova, K., Rambousek, L., Janovsky, M., Schutova, B., Hruba, L., Slamberova, R. (2009) Prenatal methamphetamine exposure affects the mesolimbic dopaminergic system and behavior in adult offspring. Int. J. Dev. Neurosci. 27(6), 525-530.

Carobrez, A. P., Bertoglio, L. J. (2005) Ethological and temporal analyses of anxiety-like behavior: the elevated plus-maze model 20 years on. Neurosci. Biobehav. Rev. 29(8), 1193-1205.

Fernández Espejo, E. (1997) Structure of the mouse behaviour on the elevated plus-maze test of anxiety. Behav. Brain Res. 86(1), 105-112.

File, S. E. (1993) The interplay of learning and anxiety in the elevated plus-maze. Behav. Brain Res. 58(1-2), 199-202. 
File, S. E., Hyde, J. R. (1978) Can social interaction be used to measure anxiety? Br. J. Pharmacol. 62(1), 19-24.

Gerlai, R., Blanchard, R. J., Blanchard, D. C. (2006) Animal models of anxiety: The ethologickal perspective. In: Contemporary Clinical Neuroscience: Transgenic and Konckout Models of Neuropsychiatric Disorders. Fisch, G. S., Flint, J., pp. 221-236, Humana Press, Totowa.

Geyer, M., Swerdlow, N. R. (2007) Behavioral neuroscience; Rat handling. In: Short Protocols in Neuroscience; Systems and Behavioral Methods. Crawley, J. N., Gerfen, C. R., Rogawski, M.A., Sibley, D. R., Skolnick, P., Wray, S. pp. 89-91, John Wiley and Sons, New Jersey.

Hassler, R., Wagner, A. (1975) Locomotor activity and speed of movements in relation to monoamine-acting drugs. Int. J. Neurol. 10(1-4), 80-97.

Hayase, T., Yamamoto, Y., Yamamoto, K. (2006) Behavioral effects of ketamine and toxic interactions with psychostimulants. BMC Neurosci. 7, 25.

Hogg, S. (1996) A review of the validity and variability of the elevated plus-maze as an animal model of anxiety. Pharmacol. Biochem. Behav. 54(1), 21-30.

Hyman, S. E., Malenka, R. C. (2001) Addiction and the brain: the neurobiology of compulsion and its persistence. Nat. Rev. Neurosci. 2(10), 695-703.

Kelley, A. E. (2004) Memory and addiction: shared neural circuitry and molecular mechanisms. Neuron 44(1), 161-179.

Kelley, A. E., Berridge, K. C. (2002) The neuroscience of natural rewards: relevance to addictive drugs. J. Neurosci. 22(9), 3306-3311.

Kitanaka, J., Kitanaka, N., Tatsuta, T., Morita, Y., Takemura, M. (2007) Blockade of brain histamine metabolism alters methamphetamine-induced expression pattern of stereotypy in mice via histamine $\mathrm{H} 1$ receptors. Neuroscience 147(3), 765-777.

Koob, G. F., Le Moal, M. (1997) Drug abuse: hedonic homeostatic dysregulation. Science 278(5335), 52-58.

Nesse, R. M., Berridge, K. C. (1997) Psychoactive drug use in evolutionary perspective. Science 278(5335), 63-66.

Pellow, S., Chopin, P., File, S. E., Briley, M. (1985) Validation of open:closed arm entries in an elevated plus-maze as a measure of anxiety in the rat. J. Neurosci. Methods 14(3), 149-167.

Pometlová, M., Mikulecká, A., Schutová, B., Hrubá, L., Deykun, K., Šlamberová, R. (2007) Vliv metamfetaminu na test sociálních interakcí u samců laboratorního potkana. (Effect of methamphetamine in the test of social interaction in adult male rats.) Psychiatrie 11, 94-97 (Suppl. 3). (in Czech)

Pometlová, M., Deykun, K., Mikulecká, A., Hrubá, L., Schutová, B., Šlamberová, R. (2008) Je snížení sociální interakce vyvolané nízkými dávkami metamfetaminu způsobené anxiogenním účinkem? (Is decrease of social interaction induced by low dose methamphetamine caused by anxiogenic effect?) Psychiatrie 12, 46-49 (Suppl. 3). (in Czech)

Rodgers, R. J., Cao, B. J., Dalvi, A., Holmes, A. (1997) Animal models of anxiety: an ethological perspective. Braz. J. Med. Biol. Res. 30(3), 289-304.

Slamberova, R., Mikulecka, A., Pometlova, M., Schutova, B., Hruba, L., Deykun, K. (2010) The effect of methamphetamine on social interaction of adult male rats. Behav. Brain Res. 214(2), 423-427.

Tatsuta, T., Kitanaka, N., Kitanaka, J., Morita, Y., Takemura, M. (2005) Effects of monoamine oxidase inhibitors on methamphetamine-induced stereotypy in mice and rats. Neurochem. Res. 30(11), 1377-1385.

Weiss, S. M., Wadsworth, G., Fletcher, A., Dourish, C.T. (1998) Utility of ethological analysis to overcome locomotor confounds in elevated maze models of anxiety. Neurosci. Biobehav. Rev. 23(2), 265-271.

Pometlová M.; Nohejlová-Deykun K.; Šlamberová R. 